\title{
FORCED OSCILLATIONS OF NONLINEAR HYPERBOLIC EQUATIONS WITH FUNCTIONAL ARGUMENTS
}

\author{
WEI NIAN LI AND BAO TONG CUI
}

\begin{abstract}
In this paper, sufficient conditions for the forced oscillations of hyperbolic equations with functional arguments of the form

$$
\begin{array}{r}
\frac{\partial^{2}}{\partial t^{2}} u(x, t)=a(t) \Delta u(x, t)+\sum_{i=1}^{m} a_{i}(t) \Delta u\left(x, \rho_{i}(t)\right)-\sum_{j=1}^{k} q_{j}(x, t) f_{j}\left(u\left(x, \sigma_{j}(t)\right)\right)+f(x, t), \\
(x, t) \in \Omega \times[0, \infty),
\end{array}
$$

are obtained, where $\Omega$ is a bounded domain in $R^{n}$ with piecewise smooth boundary $\partial \Omega$ and $\Delta$ is the Laplacian in Euclidean $n$-space $R^{n}$.
\end{abstract}

\section{Introduction}

Partial differential equations with functional arguments have been studied extensively for the past few years. However, only a few papers [1-6] have been published on the oscillation theory of hyperbolic equations with functional arguments. In this paper, we studey the forced oscillations of hyperbolic equations with functional arguments of the form

$$
\frac{\partial^{2}}{\partial t^{2}} u(x, t)=a(t) \Delta u(x, t)+\sum_{i=1}^{m} a_{i}(t) \Delta u\left(x, \rho_{i}(t)\right)-\sum_{j=1}^{k} q_{j}(x, t) f_{j}\left(u\left(x, \sigma_{j}(t)\right)\right)+f(x, t)
$$

$(x, t) \in \Omega \times[0, \infty) \equiv G$, where $\Omega$ is a bounded domain in $R^{n}$ with piecewise smooth boundary $\partial \Omega$ and $\Delta$ is the Laplacian in Euclidean $n$-space $R^{n}$.

Suppose that the following conditions (C) hold:

$\left(\mathrm{C}_{1}\right) a, a_{i} \in C([0, \infty) ;[0, \infty)), i=1,2, \ldots, m$;

$\left(\mathrm{C}_{2}\right) \rho_{i}, \sigma_{j} \in C([0, \infty) ; R), \lim _{t \rightarrow \infty} \rho_{i}(t)=\lim _{t \rightarrow \infty} \sigma_{j}(t)=\infty, i=1,2, \ldots, m ; j=$ $1,2, \ldots, k$

$\left(\mathrm{C}_{3}\right) q_{j} \in C(\bar{\Omega} \times[0, \infty) ;[0, \infty))$ and $q_{j}(t)=\min _{x \in \bar{\Omega}} q_{j}(x, t), j=1,2, \ldots, k, f \in C(\bar{\Omega} \times$ $[0, \infty) ; R)$;

Received October 29, 1997.

1991 Mathematics Subject Classification. 35B05, 35R10.

Key words and phrases. Nonlinear hyperbolic equation, functional argument, boundary value problem, forced oscillations. 
$\left(\mathrm{C}_{4}\right) f_{j} \in C(R, R), f_{j}$ are positive and convex in $(0, \infty)$ and $f_{j}(-u)=-f_{j}(u)$ for $u \geq 0$,
$j=1,2, \ldots, k$.

Our aim is to establish sufficient conditions under which every (classical) solution $u(x, t)$ of (1) satisfying a certain boundary condition is oscillatory on $\Omega \times[0, \infty)$ in the boundary conditions

$$
\frac{\partial}{\partial N} u(x, t)+\mu u(x, t)=0, \quad(x, t) \in \partial \Omega \times[0, \infty),
$$

where $N$ is the unit exterior normal vector to $\partial \Omega$ and $\mu(x, t)$ is a nonnegative continuous
function on $\partial \Omega \times[0, \infty)$, and

$$
u(x, t)=0, \quad(x, t) \in \partial \Omega \times[0, \infty) .
$$

In the following section 2 and section 3 the sufficient conditions are obtained for the oscillation of solutions of the problem (1), (2) and the problem (1), (3) in domain $G$. Note that the conditions for the oscillations for $f_{j}(u)=u, j=1,2, \ldots, k$, have been
obtained in the work of [5].

\section{Oscillation of Problem (1), (2)}

Theorem 2.1. Suppose that (C) hold and that

$\left(\mathrm{C}_{5}\right) f_{j}(u)$ are increasing in $(0, \infty), j=1,2, \ldots, k$;

$\left(\mathrm{C}_{6}\right)$ there exists a nonnegative oscillatory function $\eta \in C^{2}(R ;[0, \infty))$ such that $\eta^{\prime \prime}(t)=$ $\int_{\Omega} f(x, t) d x$ and $\lim _{t \rightarrow \infty} \eta(t)=0$.
every solution $u(x, t)$ of the problem (1), (2) is oscillatory in $G$ if the differential ineuqality

$$
y^{\prime \prime}(t)+\sum_{j=1}^{k} q_{j}(t) f_{j}\left(y\left(\sigma_{j}(t)\right)\right) \leq 0, \quad t \geq 0
$$

has no eventually positive solutions.

Proof. Suppose to the contrary that there is a nonoscillatory solution $u(x, t)$ of the problem (1), (2) which has no zero in $\Omega \times\left[t_{0}, \infty\right)$ for some $t_{0} \geq 0$. Without loss generality, we may assume that $u(x, t)>0$ in $\Omega \times\left[t_{0}, \infty\right)$. From $\left(\mathrm{C}_{2}\right)$ there exists a $t_{1} \geq t_{0}$ such that $u\left(x, \rho_{i}(t)\right)>0, i=1,2, \ldots, m$ and $u\left(x, \sigma_{j}(t)\right)>0, j=1,2, \ldots, k$, in $\Omega \times\left[t_{1}, \infty\right)$.

Integrating (1) with respect to $x$ over the domain $\Omega$, we have

$$
\begin{aligned}
\frac{d^{2}}{d t^{2}}\left(\int_{\Omega} u(x, t) d x\right)= & a(t) \int_{\Omega} \Delta u(x, t) d x+\sum_{i=1}^{m} a_{i}(t) \int_{\Omega} \Delta u\left(x, \rho_{i}(t)\right) d x \\
& -\sum_{j=1}^{k} \int_{\Omega} q_{j}(x, t) f_{j}\left(u\left(x, \sigma_{j}(t)\right)\right) d x+\int_{\Omega} f(x, t) d x .
\end{aligned}
$$


Green's formula yields

$$
\begin{aligned}
& \int_{\Omega} \Delta u(x, t) d x=\int_{\partial \Omega} \frac{\partial u}{\partial N} d S=-\int_{\partial \Omega} \mu u d S \leq 0, \quad t \geq t_{i} ; \\
& \int_{\Omega} \Delta u\left(x, \rho_{i}(t)\right) d x=\int_{\partial \Omega} \frac{\partial}{\partial N} u\left(x, \rho_{i}(t)\right) d S=-\int_{\partial \Omega} \mu\left(x, \rho_{i}(t)\right) u\left(x, \rho_{i}(t)\right) d S \leq 0, t \geq t_{1}, \\
& i=1,2, \ldots, m .
\end{aligned}
$$

From conditions $\left(\mathrm{C}_{3}\right),\left(\mathrm{C}_{4}\right)$ and Jensen's inequality, it follows that

$$
\begin{aligned}
& \int_{\Omega} q_{j}(x, t) f_{j}\left(u\left(x, \sigma_{j}(t)\right)\right) d x \geq q_{j}(t) \int_{\Omega} f_{j}\left(u\left(x, \sigma_{j}(t)\right)\right) d x \\
\geq & q_{j}(t) \int_{\Omega} d x \cdot f_{j}\left(\int_{\Omega} u\left(x, \sigma_{j}(t)\right) d x\left(\int_{\Omega} d x\right)^{-1}\right), \quad t \geq t_{1}, j=1,2, \ldots, k .
\end{aligned}
$$

Combining (6), (7) and (8), we obtain

$\frac{d^{2}}{d t^{2}}\left(\int_{\Omega} u(x, t) d x\right) \leq-\sum_{j=1}^{k} q_{j}(t) \int_{\Omega} d x f_{j}\left(\int_{\Omega} u\left(x, \sigma_{j}(t) d x\left(\int_{\Omega} d x\right)^{-1}\right)+\int_{\Omega} f(x, t) d x, \quad t \geq t_{i}\right.$.

Set

$$
y(t)=\frac{1}{|\Omega|}\left(\int_{\Omega} u(x, t) d x-\eta(t)\right), \quad t \geq t_{1}
$$

where $|\Omega|=\int_{\Omega} d x$. Then from (9) we obtain that

$$
y^{\prime \prime}(t) \leq-\sum_{j=1}^{k} q_{j}(t) f_{j}\left(\int_{\Omega} u\left(x, \sigma_{j}(t)\right) d x \frac{1}{|\Omega|}\right), \quad t \geq t_{1} .
$$

By (10) and $\left(\mathrm{C}_{5}\right)$, we have

$$
\begin{array}{r}
f_{j}\left(\int_{\Omega} u\left(x, \sigma_{j}(t)\right) d x \cdot \frac{1}{|\Omega|}\right)=f_{j}\left(y\left(\sigma_{j}(t)\right)+\frac{1}{|\Omega|} \eta\left(\sigma_{j}(t)\right)\right) \geq f_{j}\left(y\left(\sigma_{j}(t)\right),\right. \\
t \geq t_{1}, \quad j=1,2, \ldots, k
\end{array}
$$

Consequently, we get

$$
y^{\prime \prime}(t)+\sum_{j=1}^{k} q_{j}(t) f_{j}\left(y\left(\sigma_{j}(t)\right)\right) \leq 0, \quad t \geq t_{1}
$$

which contradicts assumption that (4) has no eventually positive solution.

In case $u(x, t)<0$, then the function $-u(x, t)$ is a positive solution of the problem

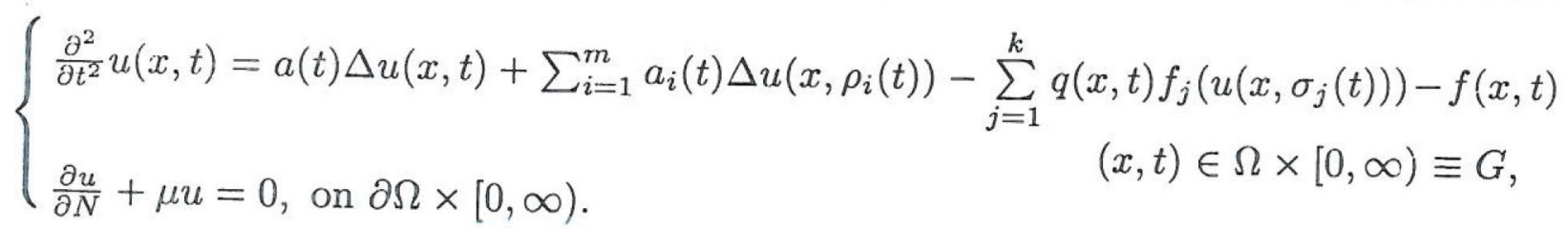


Now set $y(t)=\frac{1}{|\Omega|}\left(\int_{\Omega}-u(x, t) d x-\eta(t)\right), t \geq t_{1}$, and use an argument similar to the one used earlier to arrive at a contradiction. This completes the proof.

Lemma 2.1. ${ }^{[5]}$ Suppose that $y \in C^{2}\left(\left[t_{0}, \infty\right) ; R\right)$ and that

$$
y(t)>0, y^{\prime}(t)>0 \text { and } y^{\prime \prime}(t) \leq 0, \quad t \geq t_{0}>0 \text {. }
$$

Then for any $\lambda \in(0,1)$ there exists a number $t_{1} \geq t_{0}$, such that

$$
y(t) \geq \lambda t y^{\prime}(t) \text { for } t \geq t_{1}
$$

Theorem 2.2. Let the conditions (C) hold and that

$\left(\mathrm{C}_{7}\right)$ there exists a positive constant $M$ such that $\frac{f_{j}(u)}{u} \geq M$ for $u>0$;

$\left(\mathrm{C}_{8}\right)$ there exists an oscillatory function $\eta \in C^{2}(R ; \stackrel{R}{R})$ such that

$$
\eta^{\prime \prime}(t)=\int_{\Omega} f(x, t) d x \text { and } \lim _{t \rightarrow \infty} \eta(t)=0 .
$$

Then every solution $u(x, t)$ of the problem (1), (2) is oscillatory in $G$ if the differential inequality

$$
y^{\prime \prime}(t)+\lambda M \sum_{j=1}^{k} q_{j}(t) y\left(\sigma_{j}(t)\right) \leq 0, \quad t \geq 0
$$

has no eventually positive solutions for some $\lambda \in(0,1)$.

Proof. Suppose to the contrary that there is a nonoscillatory solution $u(x, t)$ of the problem (1), (2) which has no zero in $\Omega \times\left[t_{0}, \infty\right)$ for some $t_{0} \geq 0$. Without loss generality, we may assume that $u(x, t)>0$ in $\Omega \times\left[t_{0}, \infty\right)$. Form $\left(\mathrm{C}_{2}\right)$ there exists a $t_{1} \geq t_{0}$ such that $u\left(x, \rho_{i}(t)\right)>0, i=1,2, \ldots, m$, and $u\left(x, \sigma_{j}(t)\right)>0, j=1,2, \ldots, k$, in $\Omega \times\left[t_{1}, \infty\right)$.

Integrating (1) with respect to $x$ over the domain $\Omega$, we have

$$
\begin{aligned}
\frac{d^{2}}{d t^{2}}\left(\int_{\Omega} u(x, t) d x\right)= & a(t) \int_{\Omega} \Delta u(x, t) d x+\sum_{i=1}^{m} a_{i}(t) \int_{\Omega} \Delta u\left(x, \rho_{i}(t)\right) d x \\
& -\sum_{j=1}^{k} \int_{\Omega} q_{j}(x, t) f_{j}\left(u\left(x, \sigma_{j}(t)\right)\right) d x+\int_{\Omega} f(x, t) d x .
\end{aligned}
$$

Green's formula yields

$$
\begin{aligned}
& \int_{\Omega} \Delta u(x, t) d x=\int_{\partial \Omega} \frac{\partial u}{\partial N} d S=-\int_{\partial \Omega} \mu u d S \leq 0, \quad t \geq t_{1} \\
& \int_{\Omega} \Delta u\left(x, \rho_{i}(t)\right) d x=\int_{\partial \Omega} \frac{\partial}{\partial N} u\left(x, \rho_{i}(t)\right) d S=-\int_{\partial \Omega} \mu\left(x, \rho_{i}(t)\right) u\left(x, \rho_{i}(t)\right) d S \leq 0 \\
& t \geq t_{1}, \quad i=1,2, \ldots, m .
\end{aligned}
$$


From conditions $\left(\mathrm{C}_{3}\right),\left(\mathrm{C}_{4}\right),\left(\mathrm{C}_{7}\right)$ and Jensen's inequality, it follows that

$$
\begin{aligned}
\int_{\Omega} q_{j}(x, t) f_{j}\left(u\left(x, \sigma_{j}(t)\right)\right) d x & \geq q_{j}(t) \int_{\Omega} f_{j}\left(u\left(x, \sigma_{j}(t)\right)\right) d x \\
& \geq M q_{j}(t) \int_{\Omega} u\left(x, \sigma_{j}(t) d x, \quad t \geq t_{i}, \quad j=1,2, \ldots, k .\right.
\end{aligned}
$$

Thus we combine (17), (18) and (19) and get

$$
\frac{d^{2}}{d t^{2}}\left(\int_{\Omega} u(x, t) d x\right) \leq-M \sum_{j=1}^{k} q_{j}(t) \int_{\Omega} u\left(x, \sigma_{j}(t)\right) d x+\int_{\Omega} f(x, t) d x, \quad t \geq t_{1}
$$

Set

$$
y(t)=\int_{\Omega} u(x, t) d x-\eta(t), \quad t \geq t_{1}
$$

from (20) we obtain

$$
y^{\prime \prime}(t) \leq-M \sum_{j=1}^{k} q_{j}(t) \int_{\Omega} u\left(x, \sigma_{j}(t)\right) d x \leq 0, \quad t \geq t_{1}
$$

We claim that there is a number $t_{2} \geq t_{1}$ such that $y(t)>0, t \geq t_{2}$. In fact, if $y(t) \leq 0$ then $\int_{\Omega} u(x, t) d x \leq \eta(t)$, which is impossible in view the fact that $u(x, t)>0$ and the function $\eta$ is oscillatory. From (22) we have $y^{\prime \prime}(t) \leq 0, t \geq t_{2}$. Using the fact that $y(t)>0$ and $y^{\prime \prime}(t) \leq 0$ we have $y^{\prime}(t)>0, t \geq t_{2}$. Now, since $y$ is an increasing function and $\lim _{t \rightarrow \infty} \eta(t)=0$, it follows from (21) that there is a number $t_{3} \geq t_{2}$, by Lemma 2.1, such that

$$
\int_{\Omega} u\left(x, \sigma_{j}(t)\right) d x \geq \lambda y\left(\sigma_{j}(t)\right), \quad t \geq t_{3}, \quad j=1,2, \ldots, k
$$

Consequently, we get

$$
y^{\prime \prime}(t)+\lambda M \sum_{j=1}^{k} q_{j}(t) y\left(\sigma_{j}(t)\right) \leq 0, \quad t \geq t_{3},
$$

which contrdaicts the assumption that (15) has no eventuully positive solution.

In case $u(x, t)<0$, then the function $-u(x, t)$ is a positive solution of the problem

$$
\left\{\begin{array}{lr}
\frac{\partial^{2}}{\partial t^{2}} u(x, t)=a(t) \Delta u(x, t)+\sum_{i=1}^{m} a_{i}(t) \Delta u\left(x, \rho_{i}(t)\right)-\sum_{j=1}^{k} q(x, t) f_{j}\left(u\left(x, \sigma_{j}(t)\right)-f(x, t)\right. \\
\frac{\partial u}{\partial N}+\mu u=0, \text { on } \partial \Omega \times[0, \infty) . & (x, t) \in \Omega \times[0, \infty) \equiv G,
\end{array}\right.
$$

Now set $y(t)=\int_{\Omega}\left(-u(x, t) d x-\eta(t), t \geq t_{0}\right.$, and use arguments similar to the one used earlier to arrive at a contradiction. This completes the proof.

Theorem 2.3. Suppose that conditions $(\mathrm{C}),\left(\mathrm{C}_{7}\right)$ and $\left(\mathrm{C}_{8}\right)$ hold and that 
$\left(\mathrm{C}_{9}\right) \sigma(t)=\max _{1 \leq j \leq k}\left\{\sigma_{j}(t)\right\} \leq t, \sigma^{\prime}(t) \geq 0, t \geq t_{0}$ for some $t_{0} \geq 0$.

If there exista a $\lambda \in(0,1)$ such that

$$
\limsup _{t \rightarrow \infty} M \int_{\sigma(t)}^{t} \sum_{j=1}^{k} q_{j}(s) \sigma_{j}(s) d s>\frac{1}{\lambda^{2}}
$$

then every solution $u(x, t)$ of the problem $(1),(2)$ is oscillatory in $G$.

Proof. On the contrary let $u(x, t)$ be a nonoscillatory solution of $(1),(2)$, which we assume to be positive on $\Omega \times(0, \infty)$. Similarly to the proof of Theorem 2.2 , we can prove that the function $y$ defined by (21) satisfies the inequalities (13) and (15) for above $\lambda \in(0,1)$. By Lemma 2.1 we can choose a number $t_{1}$ sufficiently large such that $y(t) \geq \lambda t y^{\prime}(t)$ for $t \geq t_{1}$, and

$$
y\left(\sigma_{j}(t)\right) \geq \lambda \sigma_{j}(t) y^{\prime}\left(\sigma_{j}(t)\right) \text { for } t \geq t_{1}, \quad j=1,2, \ldots, k .
$$

Now, by (23) we can to get

$$
y^{\prime \prime}(t)+\lambda^{2} M \sum_{j=1}^{k} q_{j}(t) \sigma_{j}(t) y^{\prime}\left(\sigma_{j}(t)\right) \leq 0, \quad t \geq t_{1}
$$

Integrating the above inequality from $\sigma(t)$ to $t$ we have

$$
y^{\prime}(t)-y^{\prime}(\sigma(t))+\lambda^{2} M \int_{\sigma(t)}^{t} \sum_{j=1}^{k} q_{j}(s) \sigma_{j}(s) y^{\prime}\left(\sigma_{j}(s)\right) d s \leq 0, \quad t \geq t_{1} .
$$

Therefore,

$$
\lambda^{2} M \int_{\sigma(t)}^{t} \sum_{j=1}^{k} q_{j}(s) \sigma_{j}(s) y^{\prime}\left(\sigma_{j}(s)\right) d s \leq 1-\frac{y^{\prime}(t)}{y^{\prime}(\sigma(t))}<1, \quad t \geq t_{1} .
$$

And hence

$$
\limsup _{t \rightarrow \infty} M \int_{\sigma(t)}^{t} \sum_{j=1}^{k} q_{j}(s) \sigma_{j}(s) y^{\prime}\left(\sigma_{j}(s)\right) d s \leq \frac{1}{\lambda^{2}}
$$

which violates the condition (24).

The proof of the case $u(x, t)<0$ is similar and is omitted.

Corollary 2.1. In addition to conditions $(\mathrm{C})$, let $\left(C_{7}\right),\left(C_{9}\right)$ hold and suppose that $f(x, t) \equiv 0$. If

$$
\limsup _{t \rightarrow \infty} M \int_{\sigma(t)}^{t} \sum_{j=1}^{k} q_{j}(s) \sigma_{j}(s) d s>1, \text { where } \sigma(t)=\max _{1 \leq j \leq k}\left\{\sigma_{j}(t)\right\},
$$


then every solution $u(x, t)$ of the problem (1), (2) is oscillatory in $G$.

Remark 1.1. Theorem 2.2 and Theorem 2.3 improved the results of Theorem 2.1 and Theorem 2.2 from [5] and Corollary 2.1 extended the corollary 2.1 in [5].

\section{Oscilation of Problem (1),(3)}

In the domain $\Omega$ we consider the following Dirichlet problem

$$
\left\{\begin{array}{l}
\Delta u+\alpha u=0 \text { in } \Omega \\
u=0 \text { on } \partial \Omega
\end{array}\right.
$$

where $\alpha$ is a contant. It is well know $[7,8]$ that the least eigenvalue $\alpha_{0}$ of the problem (25) is positive and the corresponding eigenfuction $\varphi(x)$ is positive on $\Omega$.

Theorem. 3.1. Let the conditions $(\mathrm{C})$ and $\left(\mathrm{C}_{5}\right)$ hold and that $\left(\mathrm{C}_{10}\right)$ There exists a nonnegative oscillatory function $\eta \in C^{2}(R ;[0, \infty))$ such that

$$
\eta^{\prime \prime}(t)=\int_{\Omega} f(x, t) \varphi(x) d x \text { and } \lim _{t \rightarrow \infty} \eta(t)=0 .
$$

Then every solution $u(x, t)$ of the problem $(1),(3)$ is oscillatory in $G$ if the differential inequality

$$
y^{\prime \prime}(t)+\sum_{j=1}^{k} q_{j}(t) f_{j}\left(y\left(\sigma_{j}(t)\right)\right) \leq 0, \quad t \geq 0,
$$

has no eventually positive solutions.

Proof. Suppose to the contrary that there is a nonoscillatory solution $u(x, t)$ of the problem (1), (3), which has no zero in $\Omega \times\left[t_{0}, \infty\right)$ for some $t_{0} \geq 0$. Without loss generality, we may assume that $u(x, t)>0$ in $\Omega \times\left[t_{0}, \infty\right)$. From $\left(\mathrm{C}_{2}\right)$ there exists a $t_{1} \geq t_{0}$ such that $u\left(x, \rho_{i}(t)\right)>0, i=1,2, \ldots, m$, and $u\left(x, \sigma_{j}(t)\right)>0, j=1,2, \ldots, k$, in $\Omega \times\left[t_{1}, \infty\right)$.

Multiplying (1) by $\varphi(x)$ and integrating over $\Omega$, we obtain

$$
\begin{array}{r}
\frac{d^{2}}{d t^{2}}\left(\int_{\Omega} u(x, t) \varphi(x) d x\right)=a(t) \int_{\Omega} \Delta u(x, t) \varphi(x) d x+\sum_{j=1}^{m} a_{i}(t) \int_{\Omega} \Delta u\left(x, \rho_{i}(t)\right) \varphi(x) d x \\
-\sum_{j=1}^{k} \int_{\Omega} q_{j}(x, t) f_{j}\left(u\left(x, \sigma_{j}(t)\right)\right) \varphi(x) d x+\int_{\Omega} f(x, t) \varphi(x) d x, t \geq t_{1} .
\end{array}
$$

Using Green's formula, it follows that

$$
\begin{aligned}
& \int_{\Omega} \Delta u \varphi(x) d x=\int_{\Omega} u(x, t) \Delta \varphi(x) d x=-a_{0} \int_{\Omega} u(x, t) \varphi(x) d x \leq 0, t \geq t_{1} . \\
& \int_{\Omega} \Delta u\left(x, \rho_{i}(t)\right) \varphi(x) d x=\int_{\Omega} u\left(x, \rho_{i}(t)\right) \Delta \varphi(x) d x=-a_{0} \int_{\Omega} u\left(x, \rho_{i}(t)\right) \varphi(x) d x \leq 0, t \geq t_{1} .
\end{aligned}
$$


From conditions $\left(\mathrm{C}_{3}\right),\left(\mathrm{C}_{4}\right)$ and Jensen's inequality, it follows that

$$
\begin{aligned}
& \int_{\Omega} q_{j}(x, t) f_{j}\left(u,\left(x, \sigma_{j}(t)\right)\right) \varphi(x) d x \geq q_{j}(t) \int_{\Omega} f_{j}\left(u\left(x, \sigma_{j}(t)\right)\right) \varphi(x) d x \\
\geq & q_{j}(t) \int_{\Omega} \varphi(x) d x f_{j}\left(\int_{\Omega} u\left(x, \sigma_{j}(t)\right) \varphi(x) d x\left(\int \varphi(x) d x\right)^{-1}\right), \quad j=1,2, \ldots, k, \quad t \geq t_{1}(30)
\end{aligned}
$$

Now conbining (28), (29) and (30), we obtain

$$
\begin{aligned}
\frac{d^{2}}{d t^{2}}\left(\int_{\Omega} u(x, t) \varphi(x) d x\right) \leq & -\sum_{j=1}^{k} q_{i}(t) \int_{\Omega} \varphi(x) d x f_{j}\left(\int_{\Omega} u\left(x, \sigma_{j}(t)\right) \varphi(x) d x\left(\int_{\Omega} \varphi(x) d x\right)^{-1}\right) \\
& +\int_{\Omega} f(x, t) d x, \quad t \geq t_{1}
\end{aligned}
$$

Set

by (31), we obtain

$$
y(t)=\left(\int_{\Omega} \varphi(x) d x\right)^{-1}\left(\int_{\Omega} u(x, t) \varphi(x) d x-\eta(t)\right), \quad, t \geq t_{1},
$$

$$
y^{\prime \prime}(t) \leq-\sum_{j=1}^{k} q_{j}(t) f_{j}\left(\int_{\Omega} u\left(x, \sigma_{j}(t)\right) \varphi(x) d x\left(\int_{\Omega} \varphi(x) d x\right)^{-1}\right), \quad t \geq t_{1} .
$$

And from (32) and $\left(\mathrm{C}_{5}\right)$ we have

$$
\begin{aligned}
f_{j}\left(\int_{\Omega} u\left(x, \sigma_{j}(t)\right) \varphi(x) d x\left(\int_{\Omega} \varphi(x) d x\right)^{-1}\right) & =f_{j}\left(y\left(\sigma_{j}(t)\right)+\left(\int_{\Omega} \varphi(x) d x\right)^{-1} \cdot \eta\left(\sigma_{j}(t)\right)\right) \\
& \geq f_{j}\left(y\left(\sigma_{j}(t)\right)\right), t \geq t_{1}, j=1,2, \ldots, k .
\end{aligned}
$$

Consequently, we get

$$
y^{\prime \prime}(t)+\sum_{j=1}^{k} q_{j}(t) f_{j}\left(y\left(\sigma_{j}(t)\right)\right) \leq 0, \quad t \geq t_{1},
$$

Which contradicts the assumption that (26) has no eventually positive solution. A similar proof can be given for the case $u(x, t)<0$. This completes the proof.

Theorem 3.2. Let the conditions $(\mathrm{C})$ and $\left(\mathrm{C}_{7}\right)$ hold and that $\left(\mathrm{C}_{12}\right)$ There exists an oscillatory function $\eta \in C^{2}(R ; R)$ such that

$$
\eta^{\prime \prime}(t)=\int_{\Omega} f(x, t) \varphi(x) d x \text { and } \lim _{t \rightarrow \infty} \eta(t)=0 .
$$
Then every solution $u(x, t)$ of the problem $(1),(3)$ is oscillatory in $G$ if the differential
inequality

$$
y^{\prime \prime}(t)+\lambda M \sum_{j=1}^{k} q_{j}(t) y\left(\sigma_{j}(t)\right) \leq 0, \quad t \geq 0
$$


has no eventually positive solutions for some $\lambda \in(0,1)$.

Proof. Suppose to the contrary that there is a nonoscillatory solution $u(x, t)$ of the problem (1), (3) which has no zero in $\Omega \times\left[t_{0}, \infty\right)$ for some $t_{0} \geq 0$. Without loss generality, we may assume that $u(x, t)>0$ in $\Omega \times\left[t_{0}, \infty\right)$. From $\left(\mathrm{C}_{2}\right)$ there exists a $t_{1} \geq t_{0}$ such that $u\left(x, \rho_{i}(t)\right)>0, i=1,2, \ldots, m$ and $u\left(x, \sigma_{j}(t)\right)>0, j=1,2, \ldots, k$, in $\Omega \times\left[t_{1}, \infty\right)$.

Multiplying (1) by $\varphi(x)$ and integrating over $\Omega$, we obtain

$$
\begin{array}{r}
\frac{d^{2}}{d t^{2}}\left(\int_{\Omega} u(x, t) \varphi(x) d x\right)=a(t) \int_{\Omega} \Delta u(x, t) \varphi(x) d x+\sum_{i=1}^{m} a_{i}(t) \int_{\Omega} \Delta u\left(x, \rho_{i}(t)\right) \varphi(x) d x \\
-\sum_{j=1}^{k} \int_{\Omega} q_{j}(x, t) f_{j}\left(u\left(x, \sigma_{j}(t)\right)\right) \varphi(x) d x+\int_{\Omega} f(x, t) \varphi(x) d x
\end{array}
$$

From Green's formala it follows that

$$
\begin{aligned}
& \int_{\Omega} \Delta u \varphi(x) d x=\int_{\Omega} u(x, t) \Delta \varphi(x) d x=-\alpha_{0} \int_{\Omega} u(x, t) \varphi(x) d x \leq 0, \quad t \geq t_{i} \\
& \int_{\Omega} \Delta u\left(x, \rho_{i}(t)\right) \varphi(x) d x=\int_{\Omega} u\left(x, \rho_{i}(t)\right) \Delta \varphi(x) d x \\
& =-\alpha_{0} \int_{\Omega} u\left(x, \rho_{i}(t)\right) \varphi(x) d x \leq 0, \quad t \geq t_{1}, i=1,2, \ldots, m
\end{aligned}
$$

Moreover, from conditions $\left(\mathrm{C}_{3}\right),\left(\mathrm{C}_{4}\right)$ and $\left(\mathrm{C}_{7}\right)$ and Jensen's inequality, it follows that

$$
\begin{aligned}
& \int_{\Omega} q_{j}(x, t) f_{j}\left(u\left(x, \sigma_{j}(t)\right)\right) \varphi(x) d x \geq q_{j}(t) \int_{\Omega} f_{j}\left(u\left(x, \sigma_{j}(t)\right)\right) \varphi(x) d x \\
\geq & q_{j}(t) \int_{\Omega} \varphi(x) d x f_{j}\left(\int_{\Omega} u\left(x, \sigma_{j}(t)\right) \varphi(x) d x\left(\int_{\Omega} \varphi(x) d x\right)^{-1}\right) \\
\geq & q_{j}(t) \int_{\Omega} \varphi(x) d x M \int_{\Omega} u\left(x, \sigma_{j}(t)\right) \varphi(x) d x\left(\int_{\Omega} \varphi(x) d x\right)^{-1} \\
= & M q_{j}(t) \int_{\Omega} u\left(x, \sigma_{j}(t)\right) \varphi(x) d x, j=1,2, \ldots, k, t \geq t_{1} .
\end{aligned}
$$

Then using (37), (38) and (39), we obtain

$$
\frac{d^{2}}{d t^{2}}\left(\int_{\Omega} u(x, t) \varphi(x) d x\right) \leq-M \sum_{j=1}^{k} q_{j}(t) \int_{\Omega} u\left(x, \sigma_{j}(t)\right) \varphi(x) d x+\int_{\Omega} f(x, t) \varphi(x) d x
$$

Set

$$
y(t)=\int_{\Omega} u(x, t) \varphi(x) d x-\eta(t), \quad t \geq t_{1},
$$


by (40) we obtain

$$
y^{\prime \prime}(t) \leq-M \sum_{j=1}^{k} q_{j}(t) \int_{\Omega} u\left(x, \sigma_{j}(t)\right) \varphi(x) d x, \quad t \geq t_{1} .
$$

We note that $\int_{\Omega} u(x, t) \varphi(x) d x>0$, hence as in the proof of Theorem 2.2 we have

$$
y(t)>0, \quad t \geq t_{2} \geq t_{1}
$$

and by (42) we have

$$
y^{\prime \prime}(t) \leq 0, \quad t \geq t_{1}
$$

Form (43) and (44) it follows that $y^{\prime}(t)>0, t \geq t_{1}$. Since $y$ is an increasing function and $\lim _{t \rightarrow \infty} y(t)=0$, we conclude from $\int_{\Omega} u(x, t) \varphi(x) d x=y(t)+\eta(t)$ that there exists a number $t_{3} \geq t_{2}$ such that the following inequalities hold

$$
\begin{aligned}
& \int_{\Omega} u(x, t) \varphi(x) d x \geq \lambda y(t), \quad t \geq t_{3} \\
& \int_{\Omega} u\left(x, \sigma_{j}(t)\right) \varphi(x) d x \geq \lambda y\left(\sigma_{j}(t)\right), \quad t \geq t_{3}, j=1,2, \ldots, k .
\end{aligned}
$$

Consequently, we get

$$
y^{\prime \prime}(t)+\lambda M \sum_{j=1}^{k} q_{j}(t) y\left(\sigma_{j}(t)\right) \leq 0, \quad t \geq t_{3},
$$

which contradicts the assumption that (35) has no eventually positive solution. A similar proof can be given for the case $u(x, t)<0$. This completes the proof.

The proof of the following Theorem can be modelled on that of Theorem 3.2 and Theorem 2.3.

Theorem 3.3. Suppose that the conditions $(\mathrm{C}),\left(\mathrm{C}_{7}\right),\left(\mathrm{C}_{9}\right)$ and $\left(\mathrm{C}_{11}\right)$ hold, and that there exists a $\lambda \in(0,1)$ such that

$$
\limsup _{t \rightarrow \infty} M \int_{\sigma(t)}^{t} \sum_{j=1}^{k} q_{j}(s) \sigma_{j}(s) d s>\frac{1}{\lambda^{2}}
$$

then every solution of the problem (1), (3) is oscillatory in $G$. If

Corollary 3.1. Let conditions $(\mathrm{C}),\left(\mathrm{C}_{7}\right)$ and $\left(\mathrm{C}_{9}\right)$ hold, and suppose that $f(x, t) \equiv 0$.

$$
\limsup _{t \rightarrow \infty} M \int_{\sigma(t)}^{t} \sum_{j=1}^{k} q_{j}(s) \sigma_{j}(s) d s>1,
$$

then every solution of the problem $(1),(3)$ is oscillatory in $G$. 


\section{Examples}

Following are illustrative examples.

Example 1. Consider the hyperbolic equation

$$
\begin{aligned}
u_{t t}(x, t)= & u_{x x}(x, t)+u_{x x}(x, t-3 \pi)-e^{-t} u(x, t-3 \pi)-u(x, t-4 \pi) \\
& -e^{-t}(1+\cos x) \cos t,(x, t) \in(0, \pi) \times[0, \infty),
\end{aligned}
$$

with boundary condition

$$
u_{x}(0, t)=u_{x}(\pi, t)=0, \quad t \geq 0 .
$$

Here $a(t)=1, a_{1}(t)=1, \rho_{1}(t)=t-3 \pi, q_{1}(t)=e^{-t}, q_{2}(t)=1, f_{1}(s)=s, f_{2}(s)=s$, $f(x, t)=-e^{-t}(1+\cos x) \cos t, \sigma_{1}(t)=t-3 \pi, \sigma_{2}(t)=t-4 \pi, \sigma(t)=t-3 \pi<t$. We note that

$$
\int_{\Omega} f(x, t) d x=\int_{0}^{\pi} e^{-t}(1+\cos x) \cos t d x=-\pi e^{-t} \cos t
$$

We can choose $\eta(t)=\frac{\pi}{2} e^{-t} \cos t$. It is easy to verify that all the hypothesises of Theorem 2.3 are satisfied and hence all the solutions of problem $(45),(46)$ are oscillatory. One such solution is $u(x, t)=(1+\cos x) \cos t$.

Example 2. Consider the hyperbolic equation

$$
\begin{gathered}
u_{i \dot{t}}(x, t)=u_{x x}(x, t)+2 e^{-\frac{\pi}{2}} u_{x x}\left(x, t-\frac{\pi}{2}\right)-2 e^{-\pi} u(x, t-\pi)-e^{-t} \cos t \sin x, \\
(x, t) \in(0, \pi) \times[0, \infty) .
\end{gathered}
$$

with boundary condition

$$
u(0, t)=u(\pi, t)=0, \quad t \geq 0
$$

Here $\Omega=(0, \pi), a(t)=1, a_{1}(t)=2 e^{-\frac{\pi}{2}}, \rho_{1}(t)=t-\frac{\pi}{2}, q_{1}(t)=2 e^{-\pi}, f_{1}(s)=s, \sigma(t)=$ $\sigma_{1}(t)=t-\pi<t, f(x, t)=-e^{-t} \cos t \sin x$. Moreover, the corresponding eigenvalue problem

$$
\left\{\begin{array}{l}
\Delta u+\alpha u=0, x \in(0, \pi) \\
u=0, x=0, \pi
\end{array}\right.
$$

has the eigenvalue $\alpha_{0}=1$ with the corresponding eigenfunction $\varphi(x)=\sin x>0$ on $(0, \pi)$.

We note that

$$
\int_{\Omega} f(x, t) \varphi(x) d x=-\int_{0}^{x} e^{-t} \cos t \sin ^{2} x d x=-\frac{\pi}{2} e^{-t} \cos t .
$$

Choose the function $\eta(t)=\frac{\pi}{4} e^{-t} \sin t$. Now it is easily checked that the hypothesises of Theorem 3.3 are verified. Thus all the solutions of peroblem (48), (49) are oscillatory. One such solution is $u(x, t)=e^{-t} \cos t \sin x$. 


\section{References}

[1] B. T. Cui, Oscillation theorems of hyperbolic equations with deviating arguments, Acta Sci. Math. (Szeged) 58(1993), 159-168.

[2] B. S. Lalli, Y. H. Yu and B. T. Cui, Oscillations of certain neutral differential equations with deviating arguments, Bull. Austral. Math. Soc. 46(1992), 373-380.

[3] B. S. Lalli, Y. H. Yu and B. T. Cui, Oscillation of hyperbolic equations with functional arguments, Appl. Math. Comput. 53(1993), 97-110.

[4] D. D. Bainov and B. T. Cui, Oscillation properties for damped hyperbolic equations with deviating arguments, in Proceedings of The Third International Colloquium on Differential Equations, International Science Pubishers, 1993, 23-30(Netherlands).

[5] B. S. Lalli, Y. H. Yu and B. T. Cui, Forced Oscillations of hyperbolic differential equations with deviating arguments, Indian. J. Pure Appl. Math. 25(1994), 387-397.

[6] B. T. Cui, Oscillation properties of the solutions of hyperbolice equations with deviating arguments, Demonstratio Mathematica 29(1996), 61-68.

[7] N. Yoshida, Forced oscillations of solutions of parabolic equations, Bull. Austral. Math. Soc. 36(1987), 287-294.

[8] B. T. Cui, Oscillation theroems of nonlinear parabolic equations of neutral type, Math. J. Toyama Univ. 14(1991), 112-123.

Department of Mathematics, Binzhou Normal College, Shandong 256604, China. 